\title{
Review of Identification Aids in Biology: Past, Present and Future Challenges
}

\author{
Adeyemi Ojutalayo Adeeyo ${ }^{1}$ \\ Adepoju Tunde Joseph Ogunkunle ${ }^{1}$ \\ Olugbenga Solomon Bello \\ ${ }^{1}$ Department of Pure and Applied Biology, Ladoke Akintola University of Technology \\ P.M.B 4000, Ogbomoso, Nigeria \\ ${ }^{2}$ Department of Pure and Applied Chemistry, Ladoke Akintola University of Technology \\ P.M.B 4000, Ogbomoso, Nigeria
}

Doi:10.5901/ajis.2013.v2n13p27

\begin{abstract}
Biological identification is an applied field of Systematic Biology that deals with the construction and usage of diagnostic keys. The two primary tools of identification are tables and dichotomous keys; a third one involving the use of computer is still at various level of development. This review aims at the state of art in identification and appraisal of the various identification aids as well as challenges associated. It finally presents the recent development in computer identification aids and its future challenges for evolvement of solutions in this new area of Identification as applied to the field of Biology.
\end{abstract}

Keywords: Identification, Systematic, Diagnostic keys, Dichotomous keys, Computer.

\section{Introduction}

In theory, the act of identification, which is the ability to say an unknown unit is $A$ and unlike $B$ to $Z$, involves comparison of the unknown with the known, classified, and named units, the target being the ability to say that the unknown is like $A$ (one of the knowns) and unlike $B$ to $Z$ (all other knowns); or more objectively that the unknown is A i.e giving it a name or identification tag. When the characters can be matched, the unknown will be identified with one of the knowns. If the number of characters compared is small, then the perfect match will be common. More often, when many characters are compared, it is seldom possible to find true identity and the identifier must be satisfied with a near match in which the number of divergences is small (Queen et al., 1974). In biology, an identification key is a printed or a computer-aided device that aids the identification of biological entities, such as plants, animals, fossils, microorganisms, and pollen grains. Identification keys are also used in many other scientific and technical fields to diagnose various other entities, such as diseases, soil types, minerals, or archaeological and anthropological artifacts.

All identification schemes depend on knowing a great deal about the already identified units, but the human memory can only cope with a small proportion of this knowledge,(Queen et al., 1974). Hence the need for development of identification aids.

Identification aids are systems that contain the characters of the known used for investigating and matching the characters of the unknown. When one correctly decides that an unknown belongs to the same group as a known specimen, the information stored in classification systems becomes available and applicable to the material at hand. According to Blackwelder (1967) "identification enables us to retrieve the appropriate facts from the system to be associated with 
some specimen at hand and is better described as the recovery side of taxonomy." In practice one commonly identifies by direct comparison or the use of keys and arrives at a name. It is therefore important to have good identification aid if appropriate facts for correct taxonomy are to be retrieved from our various identification systems and aids. The objective of this work is therefore to evaluate the state of art of identification and appraise the various identification aids as well as the challenges associated with each scheme. It finally presents the recent development in computer identification aids and its future challenges for evolvement of solutions in this new area of Identification as applied to the field of Biology.

In practice, the two basic aids of identification are dichotomous keys and tables. A third technique which is the computer aid was suggested by Payne (1963). This approach has been claimed not to be readily practicable over the years (Queen et al., 1974). However, David and Shaun recently reported a revolution in computer diagnostics which is expected to replace conventional keys by matrix-based computer interactive keys (David and Shaun, 2007).

\section{Traditional Identification Aids}

There are three basic methods of biological identification namely, recognition, comparison and expert determination. Recognition, according to Morse (1974), deals with expert determination in reliability. This is based on extensive, past experience of the identifier with the group in question. In some groups this is virtually impossible. Comparison involves assessment of an unknown with named specimens, photographs, illustrations or descriptions. Even though this is a reliable method, it may be time consuming or virtually impossible due to the lack of suitable materials for comparison. The reliability is, dependent on the accuracy and authenticity of the specimens, illustrations, or descriptions used in the comparison.

A third method is by expert determination. In terms of reliability or accuracy, the best method of identification is expert determination. In general the expert will have prepared treatments (monographs, revisions, synopses) of the group in question, and it is probable that the more recent manuals include the expert's concepts of taxa. Although of great reliability, this method presents problems by requiring the valuable time of experts and creating delays for identification. All this methods of identifications uses memory aids which includes those discussed below;

\subsection{Identification Using Tables}

Conn (1900) used tables with signs for characters, and recorded the characters of his unknowns on a slip of paper that he moved over the table in much the same way as a strip is moved over tables (Cowen and Steel, 1961). In 1907, Conn and his colleagues commented that 'unless the characteristics of species could be clearly and distinctly tabulated, it was almost a hopeless task trying to identify a new culture with one previously described'. Tables of characters, mostly sugar reactions, became popular and formed large and interesting sections of books such as Manual of Tropical Medicine by Castellani and Chalmers (1919). Early identification tables however fell into disrepute due to lack of standardization of symbols. Moreover, smaller number of tests is normally needed for exact match to be made feasible, such which would not plunge the identifier into exhaustive investigation. For these reasons, diagnostic tables are less easy to use with certain details included.

\subsection{Identification using dichotomous keys}

Dichotomous keys are strategic arrangement of steps of identification such that they separate between two groups of identification whose responses are opposite. It allows a progressive response until a final option is selected which gives the answer to the requested task. The usefulness of keys varies with the kind of organism to be identified. According to Kuster (1972) 
dichotomous keys may be partial in their applications. For example in a particular work it was reported by Kuster that dichotomous keys were the most useful devices for identifying Streptomyces species while on the other hand, they are less likely to be used to identify enterobacteria.

\subsection{Identification using mechanical devices}

Bits and pieces of paper, cards, plastics, wood and metal have been put together to make gadgets aimed at simplifying the identification of bacteria. Notable examples are the Microbial Identification aid of Difco Laboratories devised by C.D Graber and the Determinator of Cowan and Steel (1960). These devices enable identification from a limited number of characters provided that the machine had been so programmed for the organisms. While these aids are to some extent useful, Queen et al.,( 1974) states that they could not allow enough leeway for the exceptions that constantly occurred among biological material.

\subsection{Identification Using Punched Card Systems}

Punch card system with or without computer sorting had been developed for the identification of limited groups of bacteria. The schemes are simple, do not involve statistical assessment of associations, and can be used with equipment no more elaborate than a card punch, scissors, and a knitting needle or probe. Example of this was that of Lederberg and Lederberg in (1952). A more elaborate system was described by Schneierson and Amsterdam (1964) in which details of staining reactions, morphological and the usual physiological and biochemical characters were recorded on a card measuring $191 \times 165 \mathrm{~mm}$. Information for notching the master cards was from authoritative reference sources (Schneierson and Amsteerdam, 1964). To be practicable, card systems must be based on the most useful distinguishing characters in the descriptions of the organisms. Punch card system is as good as diagnostic tables from which they are based (Queen et al., 1974).

\subsection{Identification Using Arithmetical or Summation Systems.}

Fey (1959) worked out a scheme in which he gave a score to each character used for his identification and added the scores of all positive characters; from the total he turned to a table from which the identification could be made. In contrast, Steel (1962) tested this scheme but found fault with it. Baer and Washington (1972) introduced confirmatory test and the modification claimed an accuracy of $99 \%$ hence arithmetical identification has shown the need for a routine confirmation before accuracy can be claimed and since they depend on tables, they will not be better than the tables on which result is inferred.

\section{Computerized Identification Aids}

Computer identification has its beginning in the early seventies (Lobanov, 1999). Two stages of computer evolution have been distinguished: First, the stage of increase in diversity of computerized keys, and second, the stage of their subsequent convergence. This was due to the unequal PC possibilities of biologists in different countries, and diversity of diagnostic programs which varied from punch cards to the developed interactive keys. Computerized diagnostic systems have been reported to have many advantages over the traditional ones in terms of efficiency, accessibility for amateurs, and in identification reliability (Lobanov, 1999).

Smirnov and Goodkov (1999) developed an interactive key for identification of Gymnamoebae. The key comprised of iconographic identification tables accompanied with the most important diagnostic features for identification of genera and supra-generic taxa of amoebae and detailed species descriptions with multiple illustrations and references to related literature for sub- 
generic identification. The iconographic tables were compiled using the conception of the morphotypes of Gymnamoebae. The design and construction of the key allow identification of amoebae with the appropriate level of accuracy up to any taxonomic level or up to the morphotype, depending on the amount of available material. Another advantage of the interactive key is especially notable for the use of protozoan ecologists, who usually do not have enough material for reliable identification of Gymnamoebae using existing keys. Smirnov and Goodkov's key was published as a set of HTML-pages controlled by embedded Java-Script scenarios. It may be used in on-line mode or as a local copy. The homepage includes comprehensive help-pages with information on the principles of the key organisation, conception of the morphotypes and on the exploitation of the "Interactive guide to Gymnamoebae" (Smirnov and Goodkov, 1999).

Dianov and Lobanov (1999) described BIKEY, a system of identification for animals which is considered one of the oldest computer diagnostic systems. The first version of BIKEY software was worked out by Lobanov (1874) for primitive computers and was later improved upon and reconstructed for modern computer platforms. The subsequent BIKEY6 and BIKEY7 versions were able to use both textual descriptions and digital images - though they were developed on MS-DOS platform only, they formed the essential part of some computer keys to many groups of animals ((Dianov and Lobanov, 1999)). The keys were designed in Zoological Institute in Germany and development of the version for Windows'95/98/NT has been reported. As the previous BIKEY versions, BIKEY8 is based on the standard database file format. The most attractive part of BIKEY 8 package is its interactive dialogue identification system. User has also a possibility of finishing the identification only by image selection without character recognition that is usually required of the expert (Dianov and Lobanov, 1999).

Lobanov and Dianov used MS Excel 7, MS Access 7 and Bikey7 to construct keys to identify nematodes. The principle of identification is to filter the taxa database by character states. At every step of identification the user can choose any character. The system has built-in algorithm which minimizes the number of identification steps. At each step DB synthesizes a new sequence of characters according to their identification values. User can use any character, but the number of identification steps can increase, if DB recommendations are neglected. The system use images as identification tools. The system has been used for identification of plant parasitic nematodes to genus level and for Pratylenchidae genera to species level (Lobanov et al., 1996)

Pyankov et al., (1999) developed Data Base (DB) of quantitative characteristics of leaf mesophyll structure in more than 1000 plant species from different climatic zones inhabiting the territory of the Former Soviet Union. These consisted of 30 plant species from the Arctics - Wrangel Island; more than 100 species from Subarctics - the Polar Urals; more than 300 species from boreal and forest-steppe zones; 200 species from Central Asian and Mongolian deserts and semi deserts; and more than 300 species of high mountain plants of West and East Pamirs. DB includes the information on the main characteristics of leaf structure: area and thickness, cell and chloroplast size, chloroplast and cell amount per leaf area unit, chloroplast number per cell; some integral indexes: total surface of mesophyll cells and total surface of chloroplasts per leaf area unit. Characteristics of different tissue types (palisade, spongy for C-3 and mesophyll, bundle sheath for C-4 species) are given for the plants with different mesophyll types. DB also includes the ecobiological data of species: life form, ecobiomorph, type of ecological strategy, and chorotype. DB is constructed in MS Excel 7 and MS Access 7. It has been used in the comparative ecophysiological study for the investigation of plant adaptations to the main ecological factors, including environmental and anthropogenic stress and for the creation of functional classification of plants, identification of plant types in boreal and arcto-alpine regions for the aims of global ecological monitoring and prognosing the vegetation under climatic changes (Pyankov et al., 1999).

Neural simulator for identification of plants is one of the possible approaches for the verification of classification models example of which Ivanova et al., (1999) have developed. The data base was created according to 30 structural characteristics of photosynthetic tissues. It includes 195 plant species. Plants were divided into 3 groups according to the type of mesophyll 
symmetry: homogenous, dorsoventral, isopalisade. Neural simulator "MultiNeuron" were used for training and identification of plants from different topological groups. Training by means of neural network with tutor was done three times in groups of 97-98 random species. The results of training were used to test the rest 97-98 species. In $90 \%$ of cases the type of mesophyll was accurately determined based only on quantitative characteristics of photosynthetic tissues (Ivanova et al., 1999).

A computer identification system has been created on the basis of empty expert systemTAXEX, for the Mediterranean fishes. The system compares the parts of a specimen to one of 151 fish families. The base of the identification is system of characters. The analysis of publications and the expert knowledge are assumed as a basis of the system of characters. Identification of an object is carried out by means of dialog with the user. The pictures with possible variants of the fragment of definable organism are proposed to the user, who has to define the figure that corresponds to the object in the best way. Next question-frame is proposed according to the earlier obtained answer. In case of need, user can access the dictionary of biologic terms. This identification system gives very good results when used by unprofessional users and students and so may be used as a training system.

A Computer identification system for 74 species of Bivalvia has been developed by Chuhchin in 1997. These species belong to 5 large orders of the genus: Heterodonta, Protobranchia, Arcoida, Anysomiaria, and Desmodonta. The data included in the system are morphology, anatomy, biology, ecology, zoogeography and descriptions of all taxa. Part of the biological and ecological information is represented in a table form for each species, namely information on feeding, distribution, occurrence, zoogeographical groups, living forms, distribution on grounds and depths. An accessible glossary of the data base describes 211 terms while all the system elements are well illustrated with 190 pictures and 22 references (Chuhchin and et al., 1997)

Program SPLIT for the identification of spurges (Euphorbia) from the Altai consists of 13 species of the genus Euphorbia, distributed in Altai region. 35 attributes (shoots system, leaves, glands, fruits and others) are analyzed. Their diagnostic value is determined by its position on the scheme. Opportunity of logic removal of any attribute to optimize the scheme is offered. Identification scheme can be chosen as the strictly dichotomous key or mixed one, with polytomous steps. Data input is similar to the traditional procedure of creation. Description of attributes is placed to the special line on the screen. A taxon may be designated by number or text label. Comments (full name of taxon; geographic distribution and others) may be attached to the label. The key is produced as step-by-step protocol and the graphic scheme. The numbers of diagnostic characters are placed on the fragments of the scheme. Two lists (of active attributes and active taxa) are added to the scheme. To define a new object, it is necessary to make a new active line, to label it and define the presence (code 1 ) or absence (code 0 ) of diagnostic characters. If it is not possible to give a precise answer, variants "it is unknown" (code $U$ - unknown), "presence and absence" (code B both) and "such attribute cannot be here" (code M - missing) are available. SPLIT demo-version is distributed free on diskettes and via internet.(Baikov, 1996).

A Computer Database and Information-diagnostic System for Identification of Syntaxon Addresses of Forest Vegetation Communities in the European Russia has been developed and it consists of interconnected blocks of : (1) DB of syntaxons of levels from vegetation type to subassociation with description of syntaxons and references between hierarchical levels; authors of publications are included. (2) Lists of diagnostic species for all syntaxons. (3) DB of references. (4) Diagnostic tables. (5) DB of geobotanic releves from publications of syntaxon's authors. (6) DB of geobotanic releves by different authors. Species are taken from computer dictionary of vascular plant species of Central Russia (Zaugol'nova et al., 1995; Khanina et al., 1999) or from computer list of flora of ex-USSR by Cherepanov's (1995) nomenclature. There is information about more than 70 vegetation associations in the system now. The programme plan is to develop diagnosis block for identification of community address by comparison of real and diagnostic species lists. Use of Syntaxon program (Onipchenko and Ovchinnikov, 1992), Ecoscale program (Zaugol'nova, 
1995) and ordination methods is also proposed (Zaugol'nova et al., 1999).

In systematization and identification of taxa of the tribe Anthemideae. DataBase fields are divided into three groups. One group consists of the field with identification features. Classification of features based on the long-term investigations (Khandjian, 1993) allows identifying the plant according to its exomorphical, anatomical, kariological, biochemical, pollen and embryonic features. Group two comprises of the fields, showing geographical location of taxa and their environment. Group three comprises of the fields, showing industrial-domestic characteristics of the plants, namely the possibility of usage of different taxa in industry and daily round. In future this information will be used to solve problems of the ex-situ and in-situ protection of the tribe representatives (Khandjian and Gulnazaryan, 1999).

The system LuclD software is one component of a solution to the identification bottleneck. LuclD is designed to capture taxonomic experts' knowledge on the identification of organisms, and to allow this knowledge to be disseminated widely. LuclD is a computer-based, multi-access, interactive identification tool, which uses Windows operating systems. The LuclD system consists of a builder module that allows quick and effective encoding of key data, and a player module that allows users to perform identifications using the builder's data. LuclD provides a link between the user's knowledge of a specimen and the builder's knowledge of the taxa. In addition to the core identification function, the builder of a LuclD key may surround the data set with an unlimited variety of information files, each piece of information tied to a taxon name. Information files in LuclD may contain notes on taxonomy, relationships, ecology or economic importance, descriptions, distribution maps, images, sound files or video clips. No constraints are placed on the builder as to what type of information or topics may be covered. In this way, LuclD becomes a publication tool for much more than the raw identification data. The main aim in developing LuclD is to deliver interactive identification tools that are flexible and powerful, as well as being extremely easy to develop and use. LuclD keys can be developed using any written language. Both the builder and player programs require minimal effort to learn, and reduce time in key construction, development and use (Yeates and Thiele, 1999).

A neuro-fuzzy expert system for hypotension control which explores approximation techniques from neural networks to find the parameter of a fuzzy system has also been developed. In the system, the traditional procedure of the medical diagnosis of hypotension employed by physician is analyzed using neuro-fuzzy inference procedure. The proposed system which is self-learning and adaptive was developed in University of Benin in Nigeria and is able to handle the uncertainties often associated with the diagnosis and analysis of hypotension (Imianvan and Obi, 2012).

Computerized system for identification of some savanna tree species in Nigeria named LEASYS was developed to serve as a modern, less time-consuming and less cumbersome method of plant identification. The LEASYS system used was based on simultaneous narrowing of two classification systems that is, vertical and horizontal characters, and supplying these systems with an appropriate Boolean operator (the AND operator preferably). The system was based on leaf morphology of some savanna tree species in Nigeria. For the purpose of this study, selected plants were identified based on possession of either simple or compound leaves. The system is amenable to expansion to cater for identification of other species in the plant kingdom (Abdulrahaman et al., 2010).

A computer Bacteriovier expert system for identification of gram negative bacteria has been developed to act as a consultant to identifiers in the field of Bacteriology. A user interacts with it to identify bacteria in question by comparing isolates to standards in the database. Allocating a score in the scoring matrix, the system calculates the similarity index for the specimen and precisely identifies it on the bases of the index. The knowledge database can be updated whenever information on a new bacterium is obtained. The software generate a report about the most probable generic name of the isolate in question and its various species which are differentiated on the basis of their response to similar tests, with a short note about the genus. The relationship between the isolate and other similar genera of organisms is also generated based on their 
similarity indices. The system developed in Ladoke Akintola University of Technology Nigeria was developed using Microsoft Basic 6.0 language and run on a personal computer with a Celeron Processor (Gueguim-kana et al., 2012).

\section{The Challenges Ahead}

A consideration of the merits derivable from computerized diagnostics makes one to conclude that the systems can compete with the best traditional "paper" publications but the new development in computer identification has led to the discovery of other limitations in this emerging field which may not completely allow for the total replacement of the conventional keys by matrix-based computerized keys.

There are still problems which include reliance on a single approach per tool (e.g., elimination in the case of multi-entry keys), use of unreliable data (e.g., by dichotomous and multi-entry keys), black box aspect of some approaches (e.g., neural networks), use of unfamiliar principles (e.g., Bayesian systems), large amount of data entry needed to be used for some Identification tools (e.g., statistic-based tools), relatively slow pace of the tool compared to a printed key, large amount of work needed to create the database, the data gathered for one tool cannot be used by other tools, database not kept up to date, lack of freedom for the user who must use the characters selected by the author of the tool and lack of freedom for the user who must obey the machine among others.

\section{Conclusion}

In conclusion, it is clear that Computer Identification Systems have evolved but these systems are still with various challenges. This system, at present, will not be able to replace conventional keys until these challenges are properly addressed. This review paper has highlighted recent and future challenges of the computer system of identification. Appropriate solutions must therefore be provided towards eradication of these bottlenecks in this new area of research. These challenges are therefore new area of research that may be of interest to researchers in the boarder of Identification and Computational Biology.

\section{References}

Abdulrahaman, A. A., Asaju, I. B., Arigbede, M. O. and Oladele, F. A. (2010), Computerized system for identification of some savanna tree species in Nigeria. Journal of Horticulture and Forestry, 2,112116.

Baer, H. and Washington, L. (1972), Numerical diagnostic key for the identification of enterobacteriaceae, Appl. Microbiology, 23,108.

Baikov, K.S. (1996), Euphorbiaceae. Florasibiriae. 10, 38-58

Blackwelder, R.E, (1967), Taxonomy, A text and reference book, John Wiley and sons. New York.

Castellani A., and Chalmers A.J., (1919), Manual of tropical medicine, edn3, London, Baillere, Tindall and Cox.

Chuhchin, V. D., Cherkasova, M. B. Lelekov, S. G. and Butako, E. A. (1997), Determinator of Gastropoda of the Black Sea, IBSS, NASU, Sevastopol, $127 \mathrm{p}$.

Conn, H.W. (1900), Classification of diary bacteria. Twelfth Ann. Rep. Storrs Agric. Exp. Sta., Storrs, Conn. 1899, 13.

Cowan, S.T. and Steel, K.J.(1960), A device for the identification of microorganisms. Lancet, I, 1172.

Cowan, S.T. and Steel, K.J . (1961), Diagnostic tables for the common medical bacteria. J. Hyg., Camb, $59,357$.

David, E. W. and Shaun, W. (2007), Keys and the crisis in taxonomy extinction or reinvention? Annual review of entomology, 52, 193-208.

Dianov, M. B. and Lobanov, A. L. (1999), Biological diagnostic system BIKEY8 for windows, International 
Symposium "Data Retrieval Systems in Biodiversity Research", St. Petersburg, Russia. 22 - 28 May. Diederich, J., Fortuner, R. and Milton, J. (1998), A General structure for biological databases, Plant pathology and biodiversity, 47-58.

Fey, H. (1959), Differenzierungsschema fur gram negative aerobe stabchen, Schweiz. Z. allg. Path. Bakt., $22,641$.

Gueguim-Kana, E.B., Adeeyo, A.O., Ogunkunle A.T.J. (2012), Expert system for identification of chemoheterotrophic enterobacteria, coccobaccilli, cocci and non-sporing rods gram negative bacteria. New clues in Sciences, 2,68-72.

Imianvan, A.A. and Obi, J.C. (2012), Cognitive Neuro-Fuzzy Expert System for Hypotension Control. Computer Engineering and Intelligent Systems, 3, 21-32.

I vanova, L., Zhukov, I. and Pyankov, V. (1999), Use of the computer neural network for the identification of plants from different biological groups based on structural characteristics of photosynthetic apparatus, International Symposium "Data Retrieval Systems in Biodiversity Research", St. Petersburg, Russia. 22 - 28 May.

Khandjian, N., Gulnazaryan, G. (1999), Data Base of the Caucasian representatives of tribe Anthemideae Cass. International Symposium "Data Retrieval Systems in

Biodiversity Research" St. Petersburg, Russia. 22 - 28 May.

Kuster, E. (1972), Simple working key for the classification and identification of named taxa included in the international Streptomyces project, Int. J. Syst. Bact., 12, 139.

Lederberg, J. and Lederberg, E.M. (1952), Replica plating and indirect selection of bacterial mutants, J. bact., 63, 399.

Lobanov, A. L. (1999), Computerized identification systems in zoology and botany - present state and perspectives, International Symposium "Data Retrieval Systems in Biodiversity Research" "St. Petersburg, Russia. 22 - 28 May.

Lobanov, A.L., Stephanjants, S.D. and Dianov, M.B. (1996), Dialogue computer system as applied to diagnostics of Cnidaria. Advances in hydrozoan biology. 60 (1): 211-220.

Morse(1974).http://www.jstor.org/pss/1218086.

Pankhurst, R.J . and Aitchison, R.R. (1975), An on-line identification program, Biological identification with computers, Academic Press, London and New York, pp. 181-185.

Payne, L.C. (1963), Towards medical automation. Wld med. Electron, 2, 6.

Pyankov, V., Ivanova, I., Kondratchouk, A., Ivanov, I. and Dzubenko, O. (1999), Data base on quantitative characteristics of leaf mesophyll structure in plants of different climatic zones. International Symposium "Data Retrieval Systems in Biodiversity Research", St. Petersburg, Russia. 22 - 28 May.

Queen, C. (1974). Diagnostic table for the common medical bacteria, $2^{\text {nd }}$ edition, University printing press, Cambridge.

Schneierson, S.S. and Amsterdam, D. (1964), A punch card system for the identification of bacteria. American Journal of Clinical Pathology, 42, 328.

Smirnov, A. V. and Goodkov, A. V. (1999),WWW-homepage, "Interactive guide to gymnamoebae". International Symposium "Data Retrieval Systems in Biodiversity Research", St. Petersburg, Russia. 22 - 28 May.

Steel, K.J . (1962), The practice of bacterial identification. Symp. Soc. Gen. Microbiol, 12, 405

Yeates, D. K. and Thiele, K. (1999), LuclD: identification tools for the biosphere. International Symposium "Data Retrieval Systems in Biodiversity Research", St. Petersburg, Russia. 22 - 28 May.

Zaugol'nova, L., Khanina, I. and Glukhova, E. (1999), Development of data base and informationdiagnostic system for identification of syntaxon addresses of forest vegetation communities in the European Russia, International Symposium "Data Retrieval Systems in Biodiversity Research", St. Petersburg, Russia. 22 - 28 May. 